\title{
The Error of the Crank-Nicolson Method for Linear Parabolic Equations with a Derivative Boundary Condition
}

\author{
OLE ØSTERBY \\ Department of Computer Science, Aarhus University \\ DK 8000 Aarhus C, Denmark
}

\begin{abstract}
The accuracy of finite difference formulae for partial differential equations is usually characterized by the approximation order of the local truncation error as determined by Taylor expansions. Thus the local truncation error of the Crank-Nicolson formula is $O\left(h^{2}+k^{2}\right)$ and this carries over to the global error in case of Dirichlet boundary conditions, but not if a derivative boundary condition is approximated by a first order formula. We study the pointwise global error on the form $h c(t, x)+k d(t, x)+h^{2} f(t, x)+k^{2} g(t, x)+\cdots$ and show that the auxiliary functions, $c, d, f, g$ are solutions to related parabolic problems. We also present a method based on the computed numbers on refined grids to determine the order of the error and the dominant terms in the error expansion. The techniques are demonstrated on two examples.
\end{abstract}

Keywords - Parabolic Equations, Difference Formulas, Global Error.

MSC - 65M12

\section{Introduction}

It is well known that the local truncation error of the explicit method and of the fully implicit method [4] for linear parabolic equations is $O\left(h^{2}+k\right)$ and of the Crank-Nicolson method [3] is $O\left(h^{2}+k^{2}\right)$ ([6], p. 119f). It is also well known that this order of approximation carries over to the global error in case of Dirichlet boundary conditions ([6], p. $63 \mathrm{f})$. The discretization of a derivative boundary condition using a first order formula will however influence the global error as shown in [1] for the explicit method.

We shall study the form of the pointwise error for the Crank-Nicolson formula on a linear parabolic equation and various discretizations of a derivative boundary condition and show that a formula which is first order in $h$ will influence the overall accuracy of the method to being only first order in $h$.

We shall pursue our investigations along two different tracks. Assuming that the global error can be expressed on the form $h c(t, x)+k d(t, x)+h^{2} f(t, x)+k^{2} g(t, x)+\cdots$ we shall 
present parabolic initial-boundary value problems for the functions in the leading terms of the error expansions, and we shall use a Richardson-like technique to reveal the (practical) order of approximation and to give approximate values of the leading auxiliary functions at selected grid points.

\section{The problem and the method}

We shall study the parabolic equation

$$
u_{t}=a u_{x x}+b u_{x}+\kappa u+\nu, \quad t \geq 0,0 \leq x \leq A
$$

where $A$ is a positive constant and where $a, b, \kappa$, and $\nu$ may depend on $t$ and $x$. The side conditions include an initial condition

$$
u(0, x)=u_{0}(x), \quad 0 \leq x \leq A
$$

where $u_{0}(x)$ is a given function of $x$, and two boundary conditions

$$
\begin{aligned}
\alpha u(t, 0)-\beta u_{x}(t, 0) & =\gamma, & & t \geq 0 \\
u(t, A) & =\delta, & & t \geq 0
\end{aligned}
$$

where $\alpha, \beta, \gamma$, and $\delta$ may depend on $t$. We have chosen a Dirichlet condition at one end for simplicity. If the coefficients are sufficiently smooth the solution $u(t, x)$ will be suitably differentiable. In our derivation we shall require $u$ to have four continuous derivatives.

For the finite difference solution we introduce a grid with step sizes $\Delta t=k$ and $\Delta x=h=$ $A / M$ where $M$ is a positive integer, and also the difference operators (in the $x$-direction):

$$
\begin{aligned}
\mu \delta v_{n, m} & =\frac{v_{n, m+1}-v_{n, m-1}}{2 h} \\
\delta^{2} v_{n, m} & =\frac{v_{n, m+1}-2 v_{n, m}+v_{n, m-1}}{h^{2}}
\end{aligned}
$$

We then compute approximate values

$v_{n, m} \approx u(n k, m h)$ for $n=0,1, \ldots$, and $m=1,2, \ldots, M-1$

using the Crank-Nicolson formula:

$$
\begin{aligned}
\frac{v_{n+1, m}-v_{n, m}}{k}= & \frac{a}{2}\left\{\delta^{2} v_{n+1, m}+\delta^{2} v_{n, m}\right\}+\frac{b}{2}\left\{\mu \delta v_{n+1, m}+\mu \delta v_{n, m}\right\} \\
& +\frac{\kappa}{2}\left\{v_{n+1, m}+v_{n, m}\right\}+\nu
\end{aligned}
$$

which is known to be second order accurate in both $k$ and $h$. If $a, b, \kappa$, and $\nu$ depend on $t$ and $x$ they should be evaluated at $t=\left(n+\frac{1}{2}\right) k, x=m h$.

Equation (7) can be rewritten using $\lambda=k / h^{2}$ to

$$
-\frac{\lambda}{2}\left(a-\frac{b h}{2}\right) v_{n+1, m-1}+\left(1+a \lambda-\frac{k \kappa}{2}\right) v_{n+1, m}-\frac{\lambda}{2}\left(a+\frac{b h}{2}\right) v_{n+1, m+1}
$$




$$
=\frac{\lambda}{2}\left(a-\frac{b h}{2}\right) v_{n, m-1}+\left(1-a \lambda+\frac{k \kappa}{2}\right) v_{n, m}+\frac{\lambda}{2}\left(a+\frac{b h}{2}\right) v_{n, m+1}+k \nu
$$

The initial condition and the second boundary condition are discretized in a straightforward manner: $v_{0, m}=u_{0}(m h), m=0,1,2, \ldots, M ; v_{n, M}=\delta(n k), n=1,2, \ldots$, and for the derivative boundary condition we shall study three discretizations based on three different finite difference approximations to the first derivative at $(t, 0)$ :

$$
\begin{aligned}
\frac{v_{n, 1}-v_{n, 0}}{h} & \text { (first order) } \\
\frac{v_{n, 1}-v_{n,-1}}{2 h} & \text { (second order, symmetric) } \\
\frac{-v_{n, 2}+4 v_{n, 1}-3 v_{n, 0}}{2 h} & \text { (second order, asymmetric) }
\end{aligned}
$$

For the sake of our analysis we shall assume that the computed solution satisfies an expansion of the form

$$
v(t, x)=u(t, x)-h c(t, x)-k d(t, x)-h^{2} f(t, x)-k^{2} g(t, x)+\cdots
$$

and we shall collect information about the auxiliary functions $c, d, f$, and $g$ which we shall assume to be suitably differentiable. Actually $v(t, x)$ is only defined on grid points $(n k, m h)$ but it can be extended (in many ways) as a differentiable function on the whole region.

\section{The auxiliary functions}

We now want to insert the expansion (12) in the difference formula (7) and use Taylor's formula to get a series in powers of $k$ and $h$. We shall therefore need the following formulas for $u$ and similarly for $c, d$, etc.

$$
\frac{u_{n+1, m}-u_{n, m}}{k}=u_{t}+\frac{1}{24} k^{2} u_{t t t}+O\left(k^{4}\right)
$$

where the derivatives on the right hand side are evaluated at the mid-point $(t, x)=((n+$ $\left.\left.\frac{1}{2}\right) k, m h\right)$.

$$
\begin{aligned}
\mu \delta u_{n, m}=\frac{u_{n, m+1}-u_{n, m-1}}{2 h} & =u_{x}+\frac{1}{6} h^{2} u_{x x x}+O\left(h^{4}\right) \\
\delta^{2} u_{n, m}=\frac{u_{n, m+1}-2 u_{n, m}+u_{n, m-1}}{h^{2}} & =u_{x x}+\frac{1}{12} h^{2} u_{x x x x}+O\left(h^{4}\right)
\end{aligned}
$$


The derivatives on the right in the above two equations are evaluated at $(t, x)=(n k, m h)$, but in the next equations we are back at the mid-point:

$$
\begin{aligned}
\frac{1}{2}\left\{u_{n+1, m}+u_{n, m}\right\} & =u+\frac{1}{8} k^{2} u_{t t}+O\left(k^{4}\right) \\
\frac{1}{2}\left\{\mu \delta u_{n+1, m}+\mu \delta u_{n, m}\right\} & =u_{x}+\frac{1}{6} h^{2} u_{x x x}+\frac{1}{8} k^{2} u_{x t t}+O\left(h^{4}+h^{2} k^{2}+k^{4}\right) \\
\frac{1}{2}\left\{\delta^{2} u_{n+1, m}+\delta^{2} u_{n, m}\right\} & =u_{x x}+\frac{1}{12} h^{2} u_{x x x x}+\frac{1}{8} k^{2} u_{x x t t}+O\left(h^{4}+h^{2} k^{2}+k^{4}\right)
\end{aligned}
$$

From the left hand side of equation (7) we now get using (12) and (13):

$$
u_{t}+\frac{1}{24} k^{2} u_{t t t}-h c_{t}-k d_{t}-h^{2} f_{t}-k^{2} g_{t}+O\left(h^{3}+h k^{2}+k^{3}\right)
$$

The right hand side is a bit more complicated:

$$
\begin{aligned}
& a\left\{u_{x x}+\frac{1}{12} h^{2} u_{x x x x}+\frac{1}{8} k^{2} u_{x x t t}-h c_{x x}-k d_{x x}-h^{2} f_{x x}-k^{2} g_{x x}\right\} \\
& +b\left\{u_{x}+\frac{1}{6} h^{2} u_{x x x}+\frac{1}{8} k^{2} u_{x t t}-h c_{x}-k d_{x}-h^{2} f_{x}-k^{2} g_{x}\right\} \\
& +\kappa\left\{u+\frac{1}{8} k^{2} u_{t t}-h c-k d-h^{2} f-k^{2} g\right\}+\nu+O\left(h^{3}+h^{2} k+h k^{2}+k^{3}\right)
\end{aligned}
$$

Equating (19) and (20) and collecting terms with various powers of $h$ and $k$ such as $1, h$, $k, h^{2}, k^{2}$ we get the following relations

$$
\begin{aligned}
u_{t} & =a u_{x x}+b u_{x}+\kappa u+\nu \\
c_{t} & =a c_{x x}+b c_{x}+\kappa c \\
d_{t} & =a d_{x x}+b d_{x}+\kappa d \\
f_{t} & =a f_{x x}+b f_{x}+\kappa f-\frac{1}{12} a u_{x x x x}-\frac{1}{6} b u_{x x x} \\
g_{t} & =a g_{x x}+b g_{x}+\kappa g+\frac{1}{24} u_{t t t}-\frac{1}{8}\left(a u_{x x t t}+b u_{x t t}+\kappa u_{t t}\right)
\end{aligned}
$$

Actually these relations need only be satisfied at the half-way grid points $t=\left(n+\frac{1}{2}\right) k$, $x=m h$, but it is natural to require them to hold in the whole region. The equation for $u$ is merely a demonstration of the fact that our difference formula is consistent with the given differential equation. We note that $c$ and $d$ should satisfy the corresponding homogeneous equation whereas $f$ and $g$ should satisfy inhomogeneous versions provided the function $u$ is four times differentiable. The last four terms in the equation for $g$ can be combined using the differential equation for $u$ to $-\frac{1}{12} u_{t t t}+\frac{1}{8} \nu_{t t}$.

We assume that the initial condition is satisfied exactly, i.e. $v_{0, m}=u(0, m h)=u_{0}(m h)$ and therefore $c(0, m h)=d(0, m h)=\cdots=0$. If we extend to the whole interval $[0, A]$ we see that it is natural to require $c(0, x)=d(0, x)=\cdots=0,0 \leq x \leq A$. Similarly the right boundary condition results in $c(t, A)=d(t, A)=\cdots=0, t \geq 0$. 


\section{A first order boundary approximation}

For the derivative boundary condition (3) we shall investigate three discretizations of the derivative term. First the simple first order approximation

$$
\alpha v_{n, 0}-\beta \frac{v_{n, 1}-v_{n, 0}}{h}=\gamma
$$

If the coefficients $\alpha, \beta$, and $\gamma$ depend on $t$ they should be evaluated at $t=n k$. Using (12) and Taylor's formula we get

$$
\begin{array}{r}
\alpha\left\{u-h c-k d-h^{2} f-k^{2} g\right\}-\beta\left\{u_{x}+\frac{1}{2} h u_{x x}+\frac{1}{6} h^{2} u_{x x x}-h c_{x}-\frac{1}{2} h^{2} c_{x x}\right. \\
\left.-k d_{x}-\frac{1}{2} h k d_{x x}-h^{2} f_{x}-k^{2} g_{x}\right\}-\gamma=O\left(h^{3}+h^{2} k+h k^{2}+k^{3}\right)
\end{array}
$$

Collecting terms with $1, h, k, h^{2}$, and $k^{2}$ as before we get

$$
\begin{aligned}
\alpha u-\beta u_{x} & =\gamma \\
\alpha c-\beta c_{x} & =-\frac{1}{2} \beta u_{x x} \\
\alpha d-\beta d_{x} & =0 \\
\alpha f-\beta f_{x} & =-\frac{1}{6} \beta u_{x x x}+\frac{1}{2} \beta c_{x x} \\
\alpha g-\beta g_{x} & =0
\end{aligned}
$$

Again the consistency of our difference approximation is reflected in relation (28) which is identical to the condition (3). Now we can collect the information we have gathered about the function $c(t, x)$ :

$$
\begin{aligned}
c_{t} & =a c_{x x}+b c_{x}+\kappa c & & t \geq 0,0 \leq x \leq A, \\
c(0, x) & =0 & & 0 \leq x \leq A, \\
\alpha c(t, 0)-\beta c_{x}(t, 0) & =-\frac{1}{2} \beta u_{x x} & & t \geq 0, \\
c(t, A) & =0 & & t \geq 0 .
\end{aligned}
$$

So $c$ must satisfy a rather similar initial-boundary value problem as $u$, homogeneous except for the derivative boundary condition. We shall return to this in the examples.

For the function $d(t, x)$ we have

$$
\begin{aligned}
d_{t} & =a d_{x x}+b d_{x}+\kappa d & & t \geq 0,0 \leq x \leq A, \\
d(0, x) & =0 & & 0 \leq x \leq A, \\
\alpha d(t, 0)-\beta d_{x}(t, 0) & =0 & & t \geq 0, \\
d(t, A) & =0 & & t \geq 0 .
\end{aligned}
$$

This is a purely homogeneous problem and as such has $d(t, x) \equiv 0$ as its (unique) solution, so we can conclude that the error is (at least) second order in $k$ as is expected from CrankNicolson. Combining with what we know about $c$ we have that the error is $O\left(h+k^{2}\right)$. 
For completeness we state the corresponding equations for $f$ and $g$ :

$$
\begin{aligned}
f_{t} & =a f_{x x}+b f_{x}+\kappa f-\frac{1}{12} a u_{x x x x}-\frac{1}{6} b u_{x x x} & & t \geq 0,0 \leq x \leq A, \\
f(0, x) & =0 & & 0 \leq x \leq A, \\
\alpha f(t, 0)-\beta f_{x}(t, 0) & =-\frac{1}{6} \beta u_{x x x}+\frac{1}{2} \beta c_{x x} & & t \geq 0, \\
f(t, A) & =0 & & t \geq 0 .
\end{aligned}
$$

and

$$
\begin{aligned}
g_{t} & =a g_{x x}+b g_{x}+\kappa g-\frac{1}{12} u_{t t t}+\frac{1}{8} \nu_{t t} & & t \geq 0,0 \leq x \leq A, \\
g(0, x) & =0 & & 0 \leq x \leq A, \\
\alpha g(t, 0)-\beta g_{x}(t, 0) & =0 & & t \geq 0, \\
g(t, A) & =0 & & t \geq 0 .
\end{aligned}
$$

The side conditions are homogeneous but the equation is not so we expect that $g(t, x) \neq 0$.

One might argue: why is there no $h k$-term in the error expansion (12). First of all we have no reason to expect such a term, but we might just for the exercise add a term $-h k e(t, x)$ in formula (12) and see what happens. Actually in formula (27) there is an $h k$-term which so far is unaccounted for. Collecting terms with $h k$ we now arrive at

$$
\begin{aligned}
e_{t} & =a e_{x x}+b e_{x}+\kappa e & & t \geq 0,0 \leq x \leq A, \\
e(0, x) & =0 & & 0 \leq x \leq A, \\
\alpha e(t, 0)-\beta e_{x}(t, 0) & =-\frac{1}{2} \beta d_{x x} & & t \geq 0, \\
e(t, A) & =0 & & t \geq 0 .
\end{aligned}
$$

We have already shown that $d(t, x) \equiv 0$ and since the equations for $e$ therefore are homogeneous we can conclude also that $e(t, x) \equiv 0$ and that there indeed is no $h k$-term in the error expansion.

\section{The symmetric second order approximation}

In the derivative boundary condition (3) we now use the symmetric second order formula (10):

$$
\alpha v_{n, 0}-\beta \frac{v_{n, 1}-v_{n,-1}}{2 h}=\gamma
$$

In order to eliminate the 'fictitious point' $v_{n,-1}$ we must also use formula (7) for $m=0$, i.e. on the left boundary but that does not change anything in our analysis. Now for the error expansion

$$
\begin{array}{r}
\alpha\left\{u-h c-k d-h^{2} f-k^{2} g\right\}-\beta\left\{u_{x}+\frac{1}{6} h^{2} u_{x x x}-h c_{x}-k d_{x}-h^{2} f_{x}-k^{2} g_{x}\right\}-\gamma \\
=O\left(h^{3}+h^{2} k+h k^{2}+k^{3}\right)
\end{array}
$$


Collecting terms with $1, h, k$, etc. as before we get

$$
\begin{aligned}
\alpha u-\beta u_{x} & =\gamma \\
\alpha c-\beta c_{x} & =0 \\
\alpha d-\beta d_{x} & =0 \\
\alpha f-\beta f_{x} & =-\frac{1}{6} \beta u_{x x x} \\
\alpha g-\beta g_{x} & =0
\end{aligned}
$$

The main difference from before is that the equations for the function $c$ are now homogeneous implying that $c(t, x) \equiv 0$ and that the error consequently is $O\left(h^{2}+k^{2}\right)$ as is customary for Crank-Nicolson.

\section{An asymmetric second order approximation}

In some cases the introduction of the fictitious point is not desirable but using formula (11) we have another way of achieving second order accuracy. The derivative boundary condition (3) now leads to:

$$
\alpha v_{n, 0}-\beta \frac{-v_{n, 2}+4 v_{n, 1}-3 v_{n, 0}}{2 h}=\gamma
$$

and the error expansion gives

$$
\begin{array}{r}
\alpha\left\{u-h c-k d-h^{2} f-k^{2} g\right\}-\beta\left\{u_{x}-\frac{1}{3} h^{2} u_{x x x}-h c_{x}-k d_{x}-h^{2} f_{x}-k^{2} g_{x}\right\}-\gamma \\
=O\left(h^{3}+h^{2} k+h k^{2}+k^{3}\right)
\end{array}
$$

Collecting terms as before we get

$$
\begin{aligned}
\alpha u-\beta u_{x} & =\gamma \\
\alpha c-\beta c_{x} & =0 \\
\alpha d-\beta d_{x} & =0 \\
\alpha f-\beta f_{x} & =\frac{1}{3} \beta u_{x x x} \\
\alpha g-\beta g_{x} & =0
\end{aligned}
$$

The only difference from the symmetric case is the factor on the right hand side in the equation for the function $f$. We conclude that also in this case $c(t, x) \equiv 0$ and that the error consequently is $O\left(h^{2}+k^{2}\right)$ as above. 


\section{Experimental determination of the order}

If one performs computations with different step sizes then a Richardson extrapolation [5] can give information on the discretization error. As before we assume that

$$
v_{1}=u-h c-k d-h^{2} f-k^{2} g+\cdots
$$

where the subscript 1 on the computed function $v$ now indicates that the computation has been performed with step sizes $k$ and $h$. We repeat the calculation with step sizes $k$ and $h / 2$ :

$$
v_{2}=u-\frac{1}{2} h c-k d-\frac{1}{4} h^{2} f-k^{2} g+\cdots
$$

By subtraction

$$
v_{2}-v_{1}=\frac{1}{2} h c+\frac{3}{4} h^{2} f+\cdots
$$

Now we repeat the calculation with step sizes $k$ and $h / 4$ :

$$
\begin{aligned}
v_{3} & =u-\frac{1}{4} h c-k d-\frac{1}{16} h^{2} f-k^{2} g+\cdots \\
v_{3}-v_{2} & =\frac{1}{4} h c+\frac{3}{16} h^{2} f+\cdots
\end{aligned}
$$

For every grid point in the coarsest grid, i.e. corresponding to $k$ and $h$ we can compute these differences and their quotient:

$$
\frac{v_{2}-v_{1}}{v_{3}-v_{2}}=2 \frac{c+\frac{3}{2} h f+\cdots}{c+\frac{3}{4} h f+\cdots}
$$

If $c(t, x) \neq 0$ and we can expect $h c$ to be the dominant term we would expect to see numbers close to 2.0 at all points indicating that the approximation is $O(h)$. On the other hand if $c(t, x) \equiv 0$ and $h^{2} f$ is the dominant term then we would expect to see numbers close to 4.0 all over the place. As it is easy to distinguish numbers close to 2.0 from numbers close to 4.0 the determination of the order of the method is pretty reliable. But one should compute these quotients for many (all) grid points because there may be (more or less) isolated points where the value deviates considerably from the otherwise accepted value, e.g. because the function $c(t, x)$ takes on a small value at the grid point in question. More about this phenomenon in the examples.

Furthermore if our investigations indicate that $c(t, x) \neq 0$ then we can get approximate values of this function from

$$
c(t, x)=\frac{4}{h}\left(v_{3}-v_{2}\right)-\frac{3}{4} h f(t, x)+\cdots
$$


by ignoring the last term. Similarly, if $c(t, x) \equiv 0$ then we can find $f(t, x)$ from

$$
f(t, x)=\frac{16}{3 h^{2}}\left(v_{3}-v_{2}\right)+\cdots
$$

Completely similar investigations can be performed w.r.t. the step size $k$ and the function $g(t, x)$ and we can thus have an experimental verification of the order of the method as well as some information on the leading terms in the error.

A further discussion of the reliability of formulas (42) - (44) will be postponed to the examples where it is easier to point out the strengths and shortcomings of this approach.

\section{An example}

We shall illustrate our techniques on a simple example involving the heat equation:

$$
u_{t}=u_{x x} \quad t \geq 0,0 \leq x \leq \pi / 2
$$

with initial condition

$$
u(0, x)=\cos x
$$

$$
0 \leq x \leq \pi / 2
$$

and boundary conditions

$$
\begin{aligned}
u_{x}(t, 0) & =0 & & t \geq 0 \\
u(t, \pi / 2) & =0 & & t \geq 0
\end{aligned}
$$

The solution is $u(t, x)=e^{-t} \cos x$.

We have solved numerically (using $h=\pi / 80$ and $k=0.025$ ) the initial-boundary value problems for $c(t, x)$ and $g(t, x)$ corresponding to the first order boundary approximation and show the results graphically in Fig. 1 and Fig. 2.

The values of $c(t, x)$ lie between 0 and 0.31 and those of $g(t, x)$ between 0 and 0.030 . With $h=\pi / 80$ the contribution to the error from $h c(t, x)$ is between 0 and 0.0013 , and with $k=0.025$ the contribution from $k^{2} g(t, x)$ is less than $0.16 \%$ of that. To reduce the truncation error it is therefore essential to reduce $h$. This will of course increase the value of $\lambda=k / h^{2}$ and consequently tend to introduce oscillations, so typical of the Crank-Nicolson process. Such oscillations are visible in Fig. 1. near $(0,0)$.

Using formula (42) and the similar one for $k$ we check the order of the method calculating the ratios on a $10 \times 10$ grid using step sizes that are four times smaller. The results are shown in Fig. 3 and Fig. 4 for $h$ and $k$ respectively. 


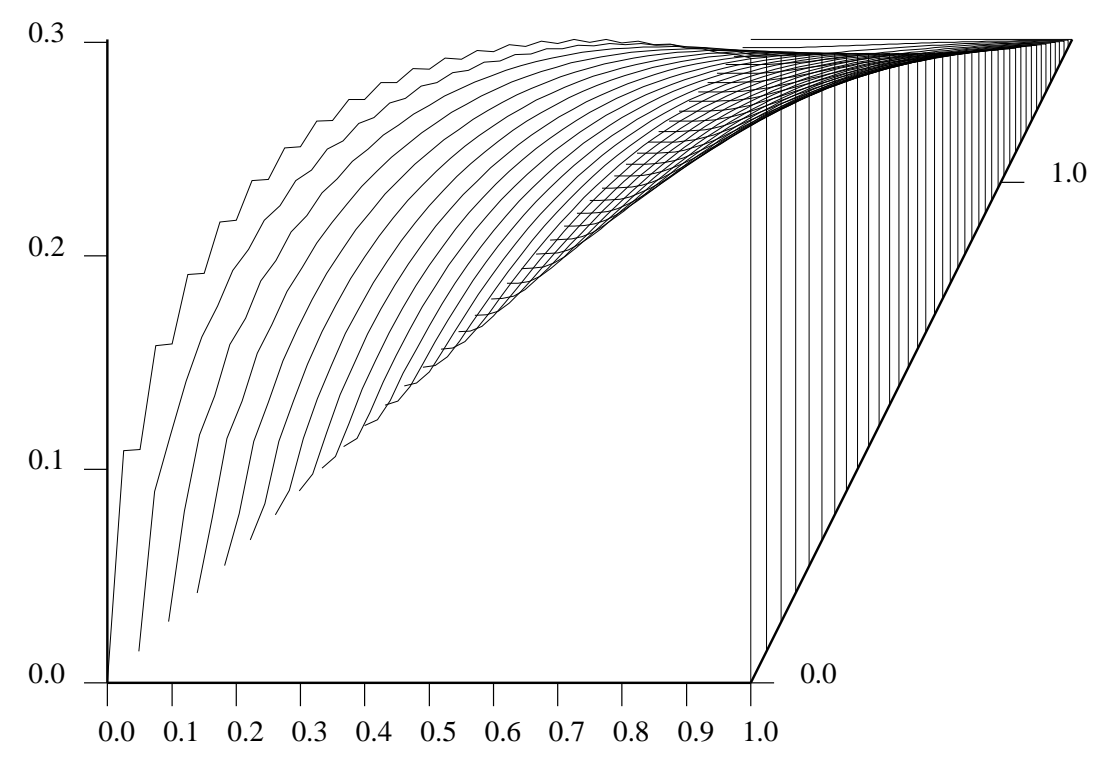

Fig. 1.

$c(t, x)$

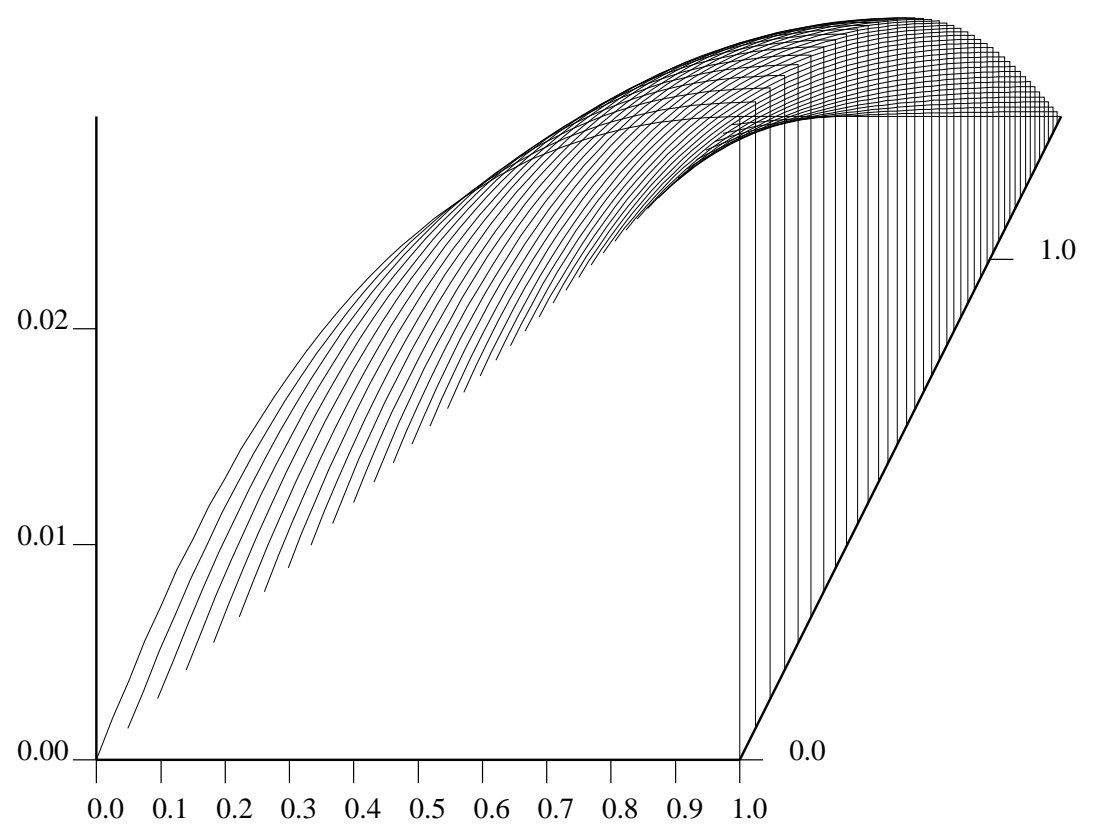

Fig. 2.

$g(t, x)$ 


$\begin{array}{lrrrrrrrrrr}\mathbf{t} \backslash \mathbf{x} & \mathbf{0 . 0 0} & \mathbf{0 . 1 6} & \mathbf{0 . 3 1} & \mathbf{0 . 4 7} & \mathbf{0 . 6 3} & \mathbf{0 . 7 9} & \mathbf{0 . 9 4} & \mathbf{1 . 1 0} & 1.26 & \mathbf{1 . 4 1} \\ \mathbf{0 . 1 0} & 2.1 & 2.1 & 2.1 & 2.1 & 2.1 & 2.1 & 2.1 & 2.1 & 1.9 & 1.6 \\ \mathbf{0 . 2 0} & 2.1 & 2.1 & 2.1 & 2.1 & 2.1 & 2.1 & 2.1 & 2.1 & 2.1 & 2.1 \\ \mathbf{0 . 3 0} & 2.1 & 2.0 & 2.0 & 2.1 & 2.1 & 2.1 & 2.1 & 2.1 & 2.1 & 2.1 \\ \mathbf{0 . 4 0} & 2.0 & 2.0 & 2.0 & 2.0 & 2.0 & 2.0 & 2.0 & 2.0 & 2.1 & 2.1 \\ 0.50 & 2.0 & 2.0 & 2.0 & 2.0 & 2.0 & 2.0 & 2.0 & 2.0 & 2.0 & 2.0 \\ 0.60 & 2.0 & 2.0 & 2.0 & 2.0 & 2.0 & 2.0 & 2.0 & 2.0 & 2.0 & 2.0 \\ 0.70 & 2.0 & 2.0 & 2.0 & 2.0 & 2.0 & 2.0 & 2.0 & 2.0 & 2.0 & 2.0 \\ 0.80 & 2.0 & 2.0 & 2.0 & 2.0 & 2.0 & 2.0 & 2.0 & 2.0 & 2.0 & 2.0 \\ 0.90 & 2.0 & 2.0 & 2.0 & 2.0 & 2.0 & 2.0 & 2.0 & 2.0 & 2.0 & 2.0 \\ 1.00 & 2.0 & 2.0 & 2.0 & 2.0 & 2.0 & 2.0 & 2.0 & 2.0 & 2.0 & 2.0\end{array}$

Fig. 3.

$h$-ratio

$\begin{array}{lrrrrrrrrrr}\mathbf{t} \backslash \mathbf{x} & \mathbf{0 . 0 0} & \mathbf{0 . 1 6} & \mathbf{0 . 3 1} & \mathbf{0 . 4 7} & \mathbf{0 . 6 3} & \mathbf{0 . 7 9} & \mathbf{0 . 9 4} & \mathbf{1 . 1 0} & \mathbf{1 . 2 6} & \mathbf{1 . 4 1} \\ \mathbf{0 . 1 0} & \mathbf{7 . 6} & -25.5 & -4.4 & -6.3 & 3.2 & \mathbf{5 . 6} & -36.6 & 4.7 & 4.4 & 4.1 \\ \mathbf{0 . 2 0} & \mathbf{7 1 . 4} & \mathbf{5 . 8} & 4.2 & 4.2 & 3.8 & 4.1 & 4.0 & 3.8 & 3.8 & 3.8 \\ \mathbf{0 . 3 0} & -11.1 & 1.7 & 4.5 & 3.9 & 4.0 & 4.0 & 4.0 & 4.0 & 4.0 & 4.0 \\ \mathbf{0 . 4 0} & -2.7 & 2.1 & 4.2 & 4.0 & 4.0 & 4.0 & 4.0 & 4.0 & 4.0 & 4.0 \\ \mathbf{0 . 5 0} & \mathbf{0 . 3} & 2.8 & 4.0 & 4.0 & 4.0 & 4.0 & 4.0 & 4.0 & 4.0 & 4.0 \\ \mathbf{0 . 6 0} & 1.8 & 3.3 & 4.0 & 4.0 & 4.0 & 4.0 & 4.0 & 4.0 & 4.0 & 4.0 \\ \mathbf{0 . 7 0} & 2.6 & 3.6 & 4.0 & 4.0 & 4.0 & 4.0 & 4.0 & 4.0 & 4.0 & 4.0 \\ \mathbf{0 . 8 0} & 3.1 & 3.8 & 4.0 & 4.0 & 4.0 & 4.0 & 4.0 & 4.0 & 4.0 & 4.0 \\ \mathbf{0 . 9 0} & 3.4 & 3.9 & 4.0 & 4.0 & 4.0 & 4.0 & 4.0 & 4.0 & 4.0 & 4.0 \\ 1.00 & 3.6 & 4.0 & 4.0 & 4.0 & 4.0 & 4.0 & 4.0 & 4.0 & 4.0 & 4.0\end{array}$

Fig. 4.

$k$-ratio

The method is clearly first order in $h$ with only few values deviating appreciably from 2.0. The picture is more confusing for $k$ where the second order is only convincing for larger values of $t$ or $x$. Negative ratios near $(0,0)$ indicate oscillating behaviour and a greater sensitivity is to be expected here since $k^{2} g(t, x)$ is so much smaller than $h c(t, x)$

The values for $c(t, x)$ as determined by (43) with $h=\pi / 80$ and $k=0.025$ agree within $7 \%$ with those obtained from solving the differential equation for $c$ and a better agreement can be obtained using smaller step sizes and/or Richardson extrapolation. The corresponding determination of $g(t, x)$ is reasonably good when $t$ and $x$ are not too close to 0 . In the regions where we have difficulty determining the order (cf. Fig. 4) we can of course have little trust in an application of formula (44) but in regions where the ratio (42) is between 
3.0 and 5.0 the agreement is within $8 \%$ with the step sizes chosen.

With the second order boundary approximations $c(t, x)$ is expected to be identically 0 , and the derivative boundary condition for $f$ reduces to $f_{x}(t, 0)=0$ since $c_{x x}=0$ and $u_{x x x}(t, 0)=e^{-t} \sin 0=0$.

The inhomogeneous term in the differential equation for $f$ is now

$$
-\frac{1}{12} a u_{x x x x}=-\frac{1}{12} e^{-t} \cos x=\frac{1}{12} u_{t t t}
$$

and we therefore see that $f(t, x)=-g(t, x)$.

For the symmetric approximation (10) the ratio (42) assumes values between 3.997 and 4.001 for the $h$-dependence and between 4.004 and 4.005 for the $k$-dependence when using $h=\pi / 20$ and $k=0.1$. These good results are due to the fact that the next terms in the error expansion (12) are $h^{4}$ and $k^{4}$ because of the symmetry and therefore interfere little. The values for $f$ and $g$ agree within $1 \%$ with each other and with the values obtained from the independent solution of the differential equation.

For the asymmetric second order boundary approximation (11) the second order in $k$ is clearly detectable on the $10 \times 10$ grid using $h=\pi / 20$ and $k=0.1$ (see Fig. 6) whereas step sizes four times smaller are needed to produce satisfactory results for $h$ (cf. Fig. 5 ). The values obtained here for $g(t, x)$ agree within $1 \%$ with those previously obtained whereas the values for $f(t, x)$ are $10-20 \%$ too small in agreement with the order being determined consistently $10-20 \%$ too small. The presence of an interfering $h^{3}$-term in the error expansion is clearly noticeable here.

\begin{tabular}{|c|c|c|c|c|c|c|c|c|c|c|}
\hline$t \backslash x$ & 0.00 & 0.16 & 0.31 & 0.47 & 0.63 & 0.79 & 0.94 & 1.10 & 1.26 & 1.41 \\
\hline 010 & 2.6 & 3.2 & 3.6 & 3.8 & 3.9 & 3.9 & 4.0 & 4.0 & 4.0 & 4.0 \\
\hline 0.20 & 3.1 & 3.4 & 3.5 & 3.7 & 3.8 & 3.8 & 3.9 & 3.9 & 3.9 & 4.0 \\
\hline 0.30 & 3.3 & 3.4 & 3.6 & 3.7 & 3.7 & 3.8 & 3.8 & 3.9 & 3.9 & 3.9 \\
\hline 0.40 & 3.3 & 3.5 & 3.6 & 3.6 & 3.7 & 3.8 & 3.8 & 3.8 & 3.8 & 3.8 \\
\hline 0.50 & 3.4 & 3.5 & 3.6 & 3.6 & 3.7 & 3.7 & 3.8 & 3.8 & 3.8 & 3.8 \\
\hline 0.60 & 3.4 & 3.5 & 3.6 & 3.6 & 3.7 & 3.7 & 3.7 & 3.8 & 3.8 & 3.8 \\
\hline 0.70 & 3.5 & 3.5 & 3.6 & 3.6 & 3.7 & 3.7 & 3.7 & 3.7 & 3.8 & 3.8 \\
\hline 0.80 & 3.5 & 3.5 & 3.6 & 3.6 & 3.7 & 3.7 & 3.7 & 3.7 & 3.7 & 3.7 \\
\hline 0.90 & 3.5 & 3.6 & 3.6 & 3.6 & 3.7 & 3.7 & 3.7 & 3.7 & 3.7 & 3.7 \\
\hline 1.00 & 3.5 & 3.6 & 3.6 & 3.6 & 3.7 & 3.7 & 3.7 & 3.7 & 3.7 & 3.7 \\
\hline
\end{tabular}

Fig. 5 .

$h$-ratio 


\begin{tabular}{|c|c|c|c|c|c|c|c|c|c|c|}
\hline$t \backslash x$ & 0.00 & 0.16 & 0.31 & 0.47 & 0.63 & 0.79 & 0.94 & 1.10 & 1.26 & 1.41 \\
\hline 0.10 & 6.3 & 5.2 & 2.4 & 3.6 & 4.4 & 4.3 & 4.1 & 4.0 & 4.0 & 3.9 \\
\hline 0.20 & 3.7 & 3.8 & 4.2 & 4.2 & 3.9 & 3.9 & 3.9 & 4.0 & 4.1 & 4.1 \\
\hline 0.30 & 4.2 & 4.1 & 3.8 & 4.0 & 4.0 & 4.0 & 4.0 & 4.0 & 4.0 & 4.0 \\
\hline 0.40 & 3.9 & 4.0 & 4.1 & 4.0 & 4.0 & 4.0 & 4.0 & 4.0 & 4.0 & 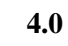 \\
\hline 0.50 & 4.0 & 4.0 & 4.0 & 4.0 & 4.0 & 4.0 & 4.0 & 4.0 & 4.0 & 4.0 \\
\hline 0.60 & 4.0 & 4.0 & 4.0 & 4.0 & 4.0 & 4.0 & 4.0 & 4.0 & 4.0 & 4.0 \\
\hline 0.70 & 4.0 & 4.0 & 4.0 & 4.0 & 4.0 & 4.0 & 4.0 & 4.0 & 4.0 & 4.0 \\
\hline 0.80 & 4.0 & 4.0 & 4.0 & 4.0 & 4.0 & 4.0 & 4.0 & 4.0 & 4.0 & 4.0 \\
\hline 0.90 & 4.0 & 4.0 & 4.0 & 4.0 & 4.0 & 4.0 & 4.0 & 4.0 & 4.0 & 4.0 \\
\hline 1.00 & 4.0 & 4.0 & 4.0 & 4.0 & 4.0 & 4.0 & 4.0 & 4.0 & 4.0 & 4.0 \\
\hline
\end{tabular}

Fig. 6.

$k$-ratio

Since $f=-g$ it is very tempting to try equal step sizes with the second order boundary approximations. Using $h=k=\pi / 80$ we can confirm that the symmetric approximation now leads to a method which is fourth order (see Fig. 7). We mention in passing that the asymmetric approximation (11) leads to a method which is third order in the common step size.

$\begin{array}{lllllllllll}\mathbf{t} \backslash \mathbf{x} & \mathbf{0 . 0 0} & \mathbf{0 . 1 6} & \mathbf{0 . 3 1} & \mathbf{0 . 4 7} & \mathbf{0 . 6 3} & \mathbf{0 . 7 9} & \mathbf{0 . 9 4} & \mathbf{1 . 1 0} & 1.26 & 1.41 \\ \mathbf{0 . 1 6} & 29.6 & 29.6 & 29.6 & 29.6 & 29.6 & 29.6 & 29.6 & 29.6 & 29.6 & 29.6 \\ \mathbf{0 . 3 1} & 27.3 & 27.3 & 27.3 & 27.3 & 27.3 & 27.3 & 27.3 & 27.3 & 27.3 & 27.3 \\ 0.47 & 25.2 & 25.2 & 25.2 & 25.2 & 25.2 & 25.2 & 25.2 & 25.2 & 25.2 & 25.2 \\ 0.63 & 23.3 & 23.3 & 23.3 & 23.3 & 23.3 & 23.3 & 23.3 & 23.3 & 23.3 & 23.3 \\ 0.79 & 21.5 & 21.5 & 21.5 & 21.5 & 21.5 & 21.5 & 21.5 & 21.5 & 21.5 & 21.5 \\ 0.94 & 19.8 & 19.8 & 19.8 & 19.8 & 19.8 & 19.8 & 19.8 & 19.8 & 19.8 & 19.8 \\ 1.10 & 18.3 & 18.3 & 18.3 & 18.3 & 18.3 & 18.3 & 18.3 & 18.3 & 18.3 & 18.3 \\ 1.26 & 16.8 & 16.8 & 16.8 & 16.8 & 16.8 & 16.8 & 16.8 & 16.8 & 16.8 & 16.8 \\ 1.41 & 15.5 & 15.5 & 15.5 & 15.5 & 15.5 & 15.5 & 15.5 & 15.5 & 15.5 & 15.5 \\ 1.00 & 14.3 & 14.3 & 14.3 & 14.3 & 14.3 & 14.3 & 14.3 & 14.3 & 14.3 & 14.3\end{array}$

Fig. 7.

$h$-ratio 


\section{$9 \quad$ An example from electrochemical kinetics}

The second example is borrowed from electrochemistry, more precisely from a model for linear potential scan voltammetry, cf. [2]. The unconditional (matrix-)stability of the CrankNicolson method may be violated if a time-dependent boundary condition is approximated in the wrong way and for this reason we analyzed several boundary approximations in that paper. We shall consider two of them here corresponding to approximations (9) and (10) and denoted F1 and $\mathrm{C} 1$ in [2].

Again we have the simple diffusion equation

$$
u_{t}=u_{x x}, \quad t \geq 0,0 \leq x \leq L
$$

where $u$ is the concentration of a chemical species. The initial concentration is

$$
u(0, x)=1, \quad 0 \leq x \leq L
$$

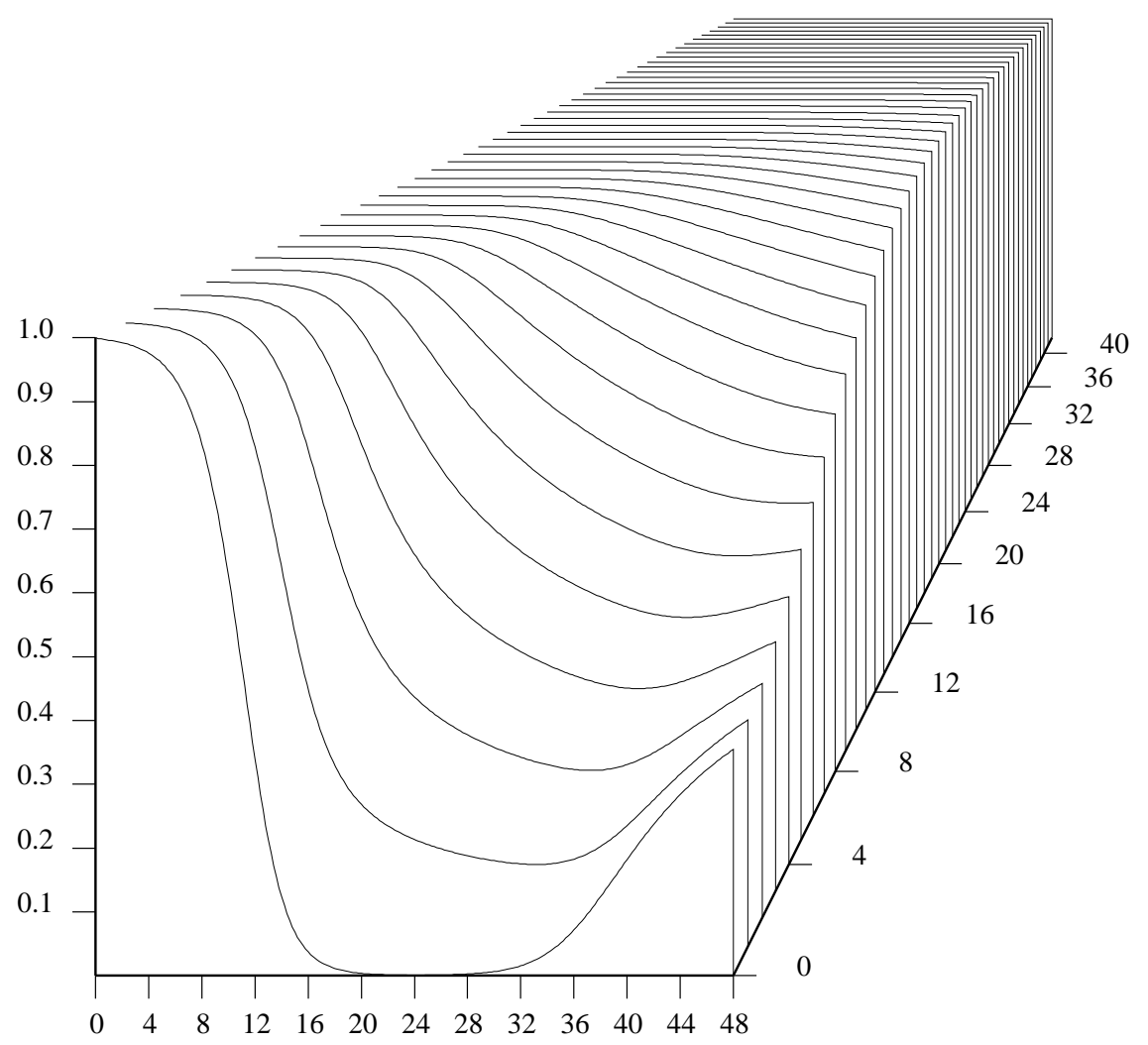

Fig. 8.

$u(t, x)$ 


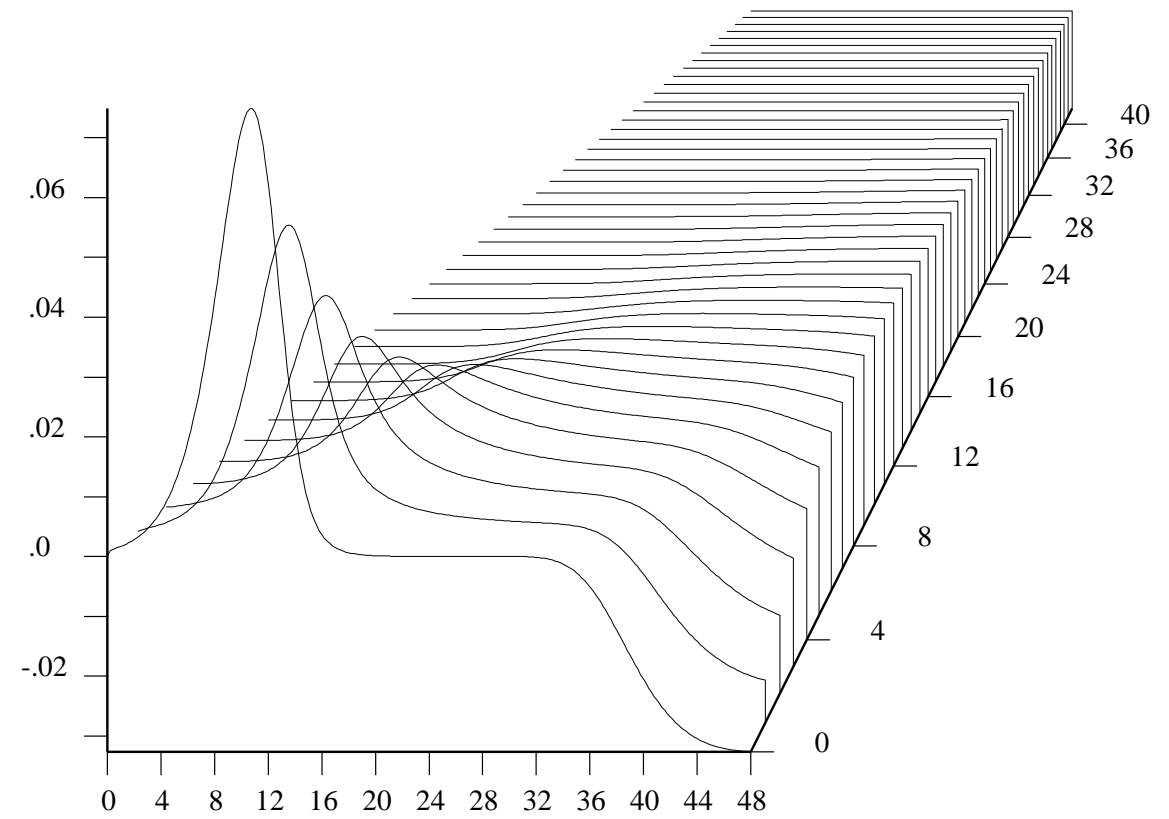

Fig. 9.

$c(t, x)$

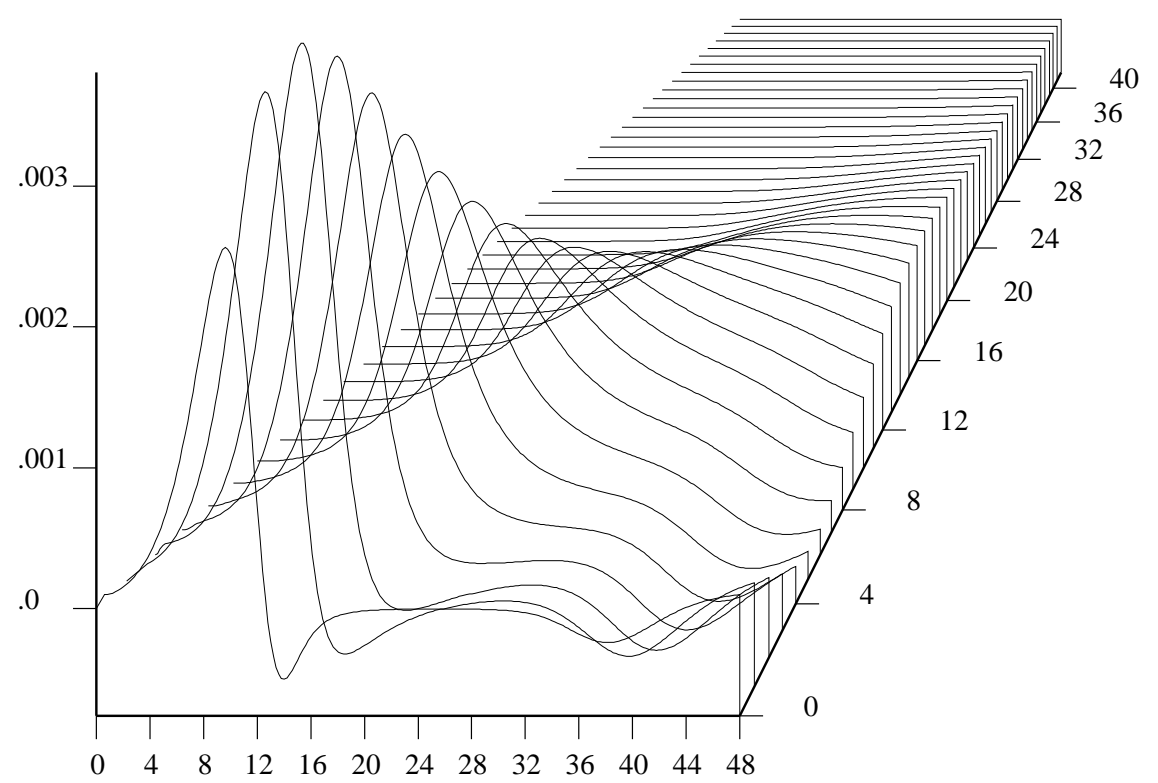

Fig. 10 .

$f(t, x)$ 


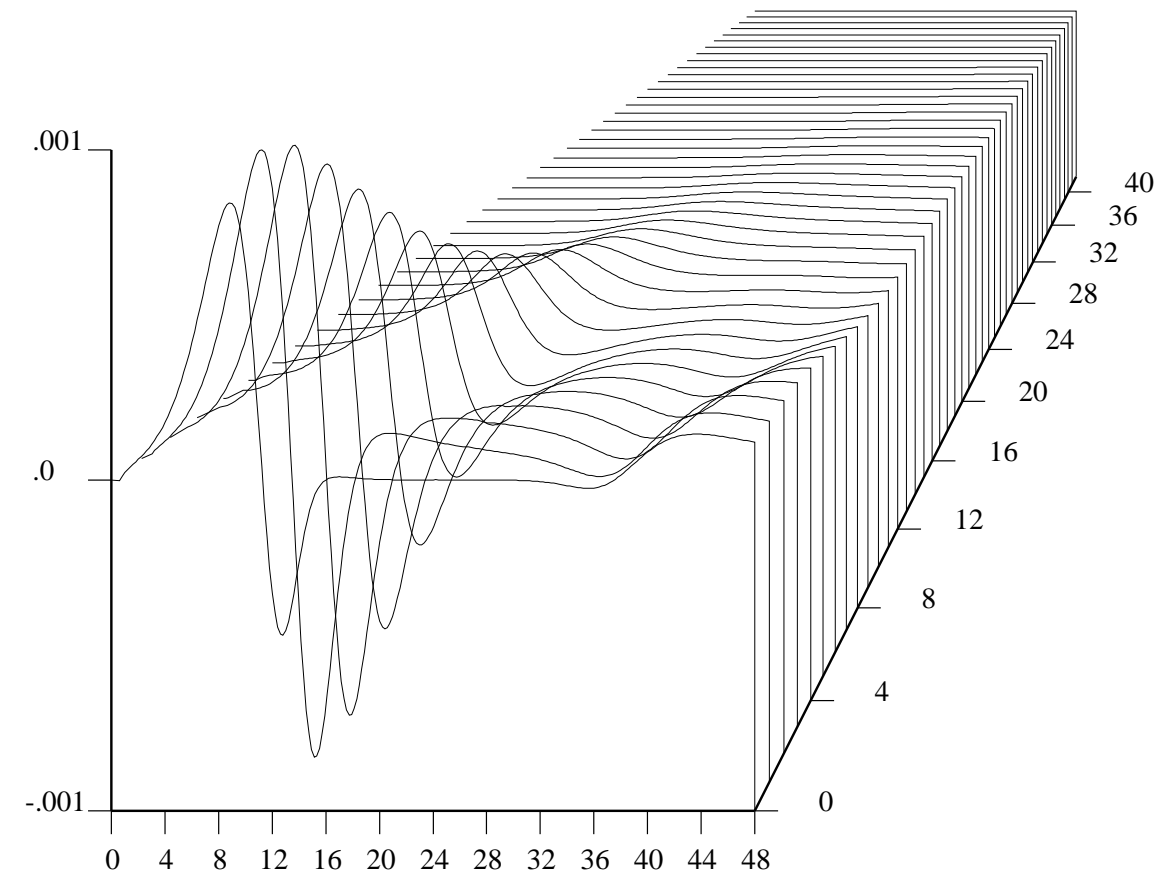

Fig. 11 . $g(t, x)$

and the boundary conditions are

$$
\begin{aligned}
\alpha(t) u(t, 0)-u_{x}(t, 0) & =0, & & t \geq 0 \\
u(t, L) & =1, & & t \geq 0
\end{aligned}
$$

where

$$
\alpha(t)= \begin{cases}\exp \left(-\frac{1}{2}(\tau-t)\right), & 0 \leq t \leq t_{s} \\ \exp \left(-\frac{1}{2}\left(\tau-2 t_{s}+t\right)\right), & t_{s}<t \leq t_{\text {max }}\end{cases}
$$

and $t_{\text {max }}=48, t_{s}=24, \tau=12, L=42 \approx 6 \sqrt{t_{\max }}$.

In Figs. 8-11 we show the concentration $u(t, x)$ and the functions $c(t, x), f(t, x)$ and $g(t, x)$ as computed numerically with step sizes $h=0.1, k=0.01$.

In Fig. 12 we show the result of applying formula (42) on part of the area in question and corresponding to the first-order approximation (9). The numbers clearly indicate an $O(h)$-approximation, and numbers significantly different from 2.0 are found only at places where $c(t, x)$ assumes small values. The corresponding numbers in Fig. 13 indicate an $O\left(k^{2}\right)$-dependence with deviations from 4.0 at small $t$-values where $g(t, x)$ is small and close to $(0,0)$ where oscillations are present. 


$\begin{array}{ccccccccccc}\mathbf{t} \backslash \mathbf{x} & \mathbf{0} & \mathbf{1} & 2 & 3 & 4 & 5 & 6 & 7 & \mathbf{8} & 9 \\ 1 & 1.8 & 2.0 & 2.1 & 2.2 & 2.3 & 2.5 & 2.6 & 2.8 & 2.9 & 2.7 \\ 2 & 1.8 & 2.0 & 2.1 & 2.1 & 2.1 & 2.2 & 2.2 & 2.3 & 2.5 & 2.6 \\ 3 & 1.9 & 2.0 & 2.0 & 2.1 & 2.1 & 2.1 & 2.1 & 2.2 & 2.2 & 2.3 \\ 4 & 2.0 & 2.0 & 2.0 & 2.1 & 2.1 & 2.1 & 2.1 & 2.1 & 2.2 & 2.2 \\ 5 & 2.0 & 2.0 & 2.0 & 2.0 & 2.1 & 2.1 & 2.1 & 2.1 & 2.1 & 2.2 \\ 6 & 2.0 & 2.0 & 2.0 & 2.0 & 2.1 & 2.1 & 2.1 & 2.1 & 2.1 & 2.1 \\ 7 & 2.0 & 2.0 & 2.0 & 2.0 & 2.1 & 2.1 & 2.1 & 2.1 & 2.1 & 2.1 \\ 8 & 2.0 & 2.0 & 2.0 & 2.0 & 2.0 & 2.1 & 2.1 & 2.1 & 2.1 & 2.1 \\ 9 & 2.0 & 2.0 & 2.0 & 2.0 & 2.0 & 2.1 & 2.1 & 2.1 & 2.1 & 2.1 \\ 10 & 2.0 & 2.0 & 2.0 & 2.0 & 2.0 & 2.1 & 2.1 & 2.1 & 2.1 & 2.1 \\ 11 & 2.0 & 2.0 & 2.0 & 2.0 & 2.0 & 2.0 & 2.1 & 2.1 & 2.1 & 2.1 \\ 12 & 2.0 & 2.0 & 2.0 & 2.0 & 2.0 & 2.0 & 2.1 & 2.1 & 2.1 & 2.1 \\ 13 & 2.0 & 2.0 & 2.0 & 2.0 & 2.0 & 2.0 & 2.0 & 2.1 & 2.1 & 2.1 \\ 14 & 2.0 & 2.0 & 2.0 & 2.0 & 2.0 & 2.0 & 2.0 & 2.0 & 2.1 & 2.1 \\ 15 & 2.0 & 2.0 & 2.0 & 2.0 & 2.0 & 2.0 & 2.0 & 2.0 & 2.1 & 2.1 \\ 16 & 2.0 & 2.0 & 2.0 & 2.0 & 2.0 & 2.0 & 2.0 & 2.0 & 2.0 & 2.1 \\ 17 & 2.0 & 2.0 & 2.0 & 2.0 & 2.0 & 2.0 & 2.0 & 2.0 & 2.0 & 2.1 \\ 18 & 1.9 & 2.0 & 2.0 & 2.0 & 2.0 & 2.0 & 2.0 & 2.0 & 2.0 & 2.0\end{array}$

Fig. 12 .

$h$-ratio

\begin{tabular}{ccccccccccc}
\hline $\mathbf{t} \backslash \mathbf{x}$ & $\mathbf{0}$ & $\mathbf{1}$ & $\mathbf{2}$ & $\mathbf{3}$ & $\mathbf{4}$ & $\mathbf{5}$ & $\mathbf{6}$ & $\mathbf{7}$ & $\mathbf{8}$ & $\mathbf{9}$ \\
1 & -42.6 & 3.2 & 4.1 & 3.7 & 4.0 & 4.0 & 4.0 & 4.2 & $\mathbf{5 . 1}$ & $\mathbf{7 . 1}$ \\
2 & -2.1 & 4.0 & 4.0 & 4.0 & 4.0 & 4.0 & 4.0 & 4.0 & 4.0 & 4.0 \\
$\mathbf{3}$ & $\mathbf{3 . 1}$ & 4.0 & 4.0 & 4.0 & 4.0 & 4.0 & 4.0 & 4.0 & 4.0 & 4.0 \\
4 & 3.9 & 4.0 & 4.0 & 4.0 & 4.0 & 4.0 & 4.0 & 4.0 & 4.0 & 4.0 \\
5 & 4.0 & 4.0 & 4.0 & 4.0 & 4.0 & 4.0 & 4.0 & 4.0 & 4.0 & 4.0 \\
$\mathbf{6}$ & 4.0 & 4.0 & 4.0 & 4.0 & 4.0 & 4.0 & 4.0 & 4.0 & 4.0 & 4.0 \\
7 & 4.0 & 4.0 & 4.0 & 4.0 & 4.0 & 4.0 & 4.0 & 4.0 & 4.0 & 4.0 \\
$\mathbf{8}$ & 4.0 & 4.0 & 4.0 & 4.0 & 4.0 & 4.0 & 4.0 & 4.0 & 4.0 & 4.0 \\
9 & 4.0 & 4.0 & 4.0 & 4.0 & 4.0 & 4.0 & 4.0 & 4.0 & 4.0 & 4.0 \\
10 & 4.0 & 4.0 & 4.0 & 4.0 & 4.0 & 4.0 & 4.0 & 4.0 & 4.0 & 4.0 \\
11 & 4.0 & 4.0 & 4.0 & 4.0 & 4.0 & 4.0 & 4.0 & 4.0 & 4.0 & 4.0 \\
12 & 4.0 & 4.0 & 4.0 & 4.0 & 4.0 & 4.0 & 4.0 & 4.0 & 4.0 & 4.0 \\
13 & 4.0 & 4.0 & 4.0 & 4.0 & 4.0 & 4.0 & 4.0 & 4.0 & 4.0 & 4.0 \\
14 & 4.0 & 4.0 & 4.0 & 4.0 & 4.0 & 4.0 & 4.0 & 4.0 & 4.0 & 4.0 \\
15 & 4.0 & 4.0 & 4.0 & 4.0 & 4.0 & 4.0 & 4.0 & 4.0 & 4.0 & 4.0 \\
16 & 4.0 & 4.0 & 4.0 & 4.0 & 4.0 & 4.0 & 4.0 & 4.0 & 4.0 & 4.0 \\
17 & 4.0 & 4.0 & 4.0 & 4.0 & 4.0 & 4.0 & 4.0 & 4.0 & 4.0 & 4.0 \\
18 & 4.0 & 4.0 & 4.0 & 4.0 & 4.0 & 4.0 & 4.0 & 4.0 & 4.0 & 4.0
\end{tabular}

Fig. 13 .

$k$-ratio 


\begin{tabular}{|c|c|c|c|c|c|c|c|c|c|c|}
\hline$t \backslash x$ & 0 & 1 & 2 & 3 & 4 & 5 & 6 & 7 & 8 & 9 \\
\hline 1 & 2.5 & 4.0 & 4.0 & 4.0 & 4.0 & 4.0 & 4.0 & 4.1 & 4.0 & 2.7 \\
\hline 2 & 1.5 & 4.0 & 4.0 & 4.0 & 4.0 & 4.0 & 4.0 & 4.0 & 4.0 & 4.0 \\
\hline 3 & 0.8 & 4.0 & 4.0 & 4.0 & 4.0 & 4.0 & 4.0 & 4.0 & 4.0 & 4.0 \\
\hline 4 & 0.1 & 4.0 & 4.0 & 4.0 & 4.0 & 4.0 & 4.0 & 4.0 & 4.0 & 4.0 \\
\hline 5 & -1.0 & 4.0 & 4.0 & 4.0 & 4.0 & 4.0 & 4.0 & 4.0 & 4.0 & 4.0 \\
\hline 6 & -3.6 & 4.0 & 4.0 & 4.0 & 4.0 & 4.0 & 4.0 & 4.0 & 4.0 & 4.0 \\
\hline 7 & -21.7 & 4.0 & 4.0 & 4.0 & 4.0 & 4.0 & 4.0 & 4.0 & 4.0 & 4.0 \\
\hline 8 & 16.4 & 4.0 & 4.0 & 4.0 & 4.0 & 4.0 & 4.0 & 4.0 & 4.0 & 4.0 \\
\hline 9 & 8.7 & 4.0 & 4.0 & 4.0 & 4.0 & 4.0 & 4.0 & 4.0 & 4.0 & 4.0 \\
\hline 10 & 7.3 & 4.0 & 4.0 & 4.0 & 4.0 & 4.0 & 4.0 & 4.0 & 4.0 & 4.0 \\
\hline 11 & 7.7 & 4.0 & 4.0 & 4.0 & 4.0 & 4.0 & 4.0 & 4.0 & 4.0 & 4.0 \\
\hline 12 & 14.4 & 4.0 & 4.0 & 4.0 & 4.0 & 4.0 & 4.0 & 4.0 & 4.0 & 4.0 \\
\hline 13 & -4.2 & 4.0 & 4.0 & 4.0 & 4.0 & 4.0 & 4.0 & 4.0 & 4.0 & 4.0 \\
\hline 14 & 0.1 & 4.0 & 4.0 & 4.0 & 4.0 & 4.0 & 4.0 & 4.0 & 4.0 & 4.0 \\
\hline 15 & 0.5 & 4.0 & 4.0 & 4.0 & 4.0 & 4.0 & 4.0 & 4.0 & 4.0 & 4.0 \\
\hline 16 & 0.5 & 4.0 & 4.0 & 4.0 & 4.0 & 4.0 & 4.0 & 4.0 & 4.0 & 4.0 \\
\hline 17 & 0.3 & 4.0 & 4.0 & 4.0 & 4.0 & 4.0 & 4.0 & 4.0 & 4.0 & 4.0 \\
\hline 18 & 0.2 & 4.0 & 4.0 & 4.0 & 4.0 & 4.0 & 4.0 & 4.0 & 4.0 & 4.0 \\
\hline
\end{tabular}

Fig. 14 .

$h$-ratio

$\begin{array}{ccccccccccc}\mathbf{t} \backslash \mathbf{x} & \mathbf{0} & \mathbf{1} & \mathbf{2} & \mathbf{3} & \mathbf{4} & \mathbf{5} & \mathbf{6} & \mathbf{7} & \mathbf{8} & \mathbf{9} \\ \mathbf{1} & \mathbf{5 4 . 7} & 2.7 & 4.0 & 3.9 & 4.0 & 4.0 & 4.0 & 4.3 & 5.2 & \mathbf{7 . 3} \\ 2 & -36.7 & 4.0 & 4.0 & 4.0 & 4.0 & 4.0 & 4.0 & 4.0 & 4.0 & 4.0 \\ 3 & -3.5 & 4.0 & 4.0 & 4.0 & 4.0 & 4.0 & 4.0 & 4.0 & 4.0 & 4.0 \\ 4 & 2.5 & 4.0 & 4.0 & 4.0 & 4.0 & 4.0 & 4.0 & 4.0 & 4.0 & 4.0 \\ \mathbf{5} & 3.7 & 4.0 & 4.0 & 4.0 & 4.0 & 4.0 & 4.0 & 4.0 & 4.0 & 4.0 \\ 6 & 3.9 & 4.0 & 4.0 & 4.0 & 4.0 & 4.0 & 4.0 & 4.0 & 4.0 & 4.0 \\ 7 & 4.0 & 4.0 & 4.0 & 4.0 & 4.0 & 4.0 & 4.0 & 4.0 & 4.0 & 4.0 \\ 8 & 4.0 & 4.0 & 4.0 & 4.0 & 4.0 & 4.0 & 4.0 & 4.0 & 4.0 & 4.0 \\ 9 & 4.0 & 4.0 & 4.0 & 4.0 & 4.0 & 4.0 & 4.0 & 4.0 & 4.0 & 4.0 \\ 10 & 4.0 & 4.0 & 4.0 & 4.0 & 4.0 & 4.0 & 4.0 & 4.0 & 4.0 & 4.0 \\ 11 & 4.0 & 4.0 & 4.0 & 4.0 & 4.0 & 4.0 & 4.0 & 4.0 & 4.0 & 4.0 \\ 12 & 4.0 & 4.0 & 3.3 & 4.0 & 4.0 & 4.0 & 4.0 & 4.0 & 4.0 & 4.0 \\ 13 & 4.0 & 4.0 & 4.0 & 4.0 & 4.0 & 4.0 & 4.0 & 4.0 & 4.0 & 4.0 \\ 14 & 4.0 & 4.0 & 4.0 & 4.0 & 4.0 & 4.0 & 4.0 & 4.0 & 4.0 & 4.0 \\ 15 & 4.0 & 4.0 & 4.0 & 4.0 & 4.0 & 4.0 & 4.0 & 4.0 & 4.0 & 4.0 \\ 16 & 4.0 & 4.0 & 4.0 & 4.0 & 4.0 & 4.0 & 4.0 & 4.0 & 4.0 & 4.0 \\ 17 & 4.0 & 4.0 & 4.0 & 4.0 & 4.0 & 4.0 & 4.0 & 4.0 & 4.0 & 4.0 \\ 18 & 4.0 & 4.0 & 4.0 & 4.0 & 4.0 & 4.0 & 4.0 & 4.0 & 4.0 & 4.0\end{array}$

Fig. 15 .

$k$-ratio 
A comparison of the $c(t, x)$-values determined from the numerical integration and from formula (43) reveal deviations less than $10 \%$ everywhere except in a small region near $(0,0)$.

Using the symmetric boundary approximation (10) we get results which are second order in $h$ as well as in $k$ as witnessed by Figs. 14 and 15 .

The values for $g(t, x)$ as computed by the formula corresponding to (44) for the two boundary approximations deviate by less than $5 \%$ in most places. The correspondence with the values from the numerical integration is not quite as good near $(0,0)$ where oscillations influence the quality of the numerical integration.

\section{References}

[1] George W. Batten. Second-Order Correct Boundary Conditions for the numerical solution of the mixed boundary problem for parabolic equations. Math. Comput., 17:405-413, 1963.

[2] Leslaw K. Bieniasz, Ole Østerby and Dieter Britz. The Effect of the Discretization of the Mixed Boundary Conditions on the Numerical Stability of the Crank-Nicolson Algorithm of Electrochemical Kinetic Simulations. to appear in Computers and Chemistry, 1997.

[3] J. Crank and P. Nicolson. A practical method for numerical integration of solution of partial differential equations of heat-conduction type. Proc. Camb. Philos. Soc., 43:50-67, 1947.

[4] P. Laasonen. Über eine Methode zur Lösung der Wärmeleitungsgleichung. Acta Math., 81:309-317, 1949.

[5] L. F. Richardson. The Deferred Approach to the Limit. Trans. Roy. Soc. London, 226:299-349, 1927.

[6] John C. Strikwerda. Finite Difference Schemes and Partial Differential Equations. Wadsworth and Brooks/Cole, Pacific Grove, CA, 1989. 